\title{
Interference of Hsa_circ_0003928 Alleviates High Glucose-Induced Cell Apoptosis and Inflammation in HK-2 Cells via miR-I5I-3p/Anxa2
}

This article was published in the following Dove Press journal: Diabetes, Metabolic Syndrome and Obesity: Targets and Therapy

\author{
Ling $A n^{1, *}$ \\ Dongde $\mathrm{Ji}^{2, *}$ \\ Wenbo Hu' \\ Jianrong Wang' \\ Xiuzhen Jin ${ }^{3}$ \\ Yunfei $\mathrm{Qu}^{4}$ \\ Ning Zhang ${ }^{5}$

\begin{abstract}
'Department of Nephrology, Qinghai Provincial People's Hospital, Xining 810007, People's Republic of China; ${ }^{2}$ Department of Gastroenterology, Qinghai Provincial People's Hospital, Xining 810007, People's Republic of China; ${ }^{3}$ Department of Nursing, Qinghai Institute of Health Sciences, Xining 810007, People's Republic of China; ${ }^{4}$ Department of Cardiovascular Surgery, Chongqing University Three Gorges Hospital, Chongqing 404000, People's Republic of China; ${ }^{5}$ Department of General Practice, Chongqing University Three Gorges Hospital, Chongqing 404000, People's Republic of China

*These authors contributed equally to this work.
\end{abstract}

Correspondence: Yunfei Qu; Ning Zhang Chongqing University Three Gorges Hospital, No. 165, Xincheng Road, Wanzhou District, Chongqing 404000, People's Republic of China Email yunfeiqu052I@I63.com; zhangnning667@163.com
Background: Diabetic nephropathy (DN) is a severe end-stage kidney disease developed from diabetes mellitus. The involvement of circular RNA (circRNAs) in the regulation of DN pathogenesis has been implied, but the underlying mechanism of DN is still lacking. This study aimed to investigate the effect of hsa_circ_0003928 on the inflammation and apoptosis of high glucose (HG)-induced renal tubular cells.

Methods: The expression of hsa_circ_0003928, miR-151-3p and Anxa2 in blood samples from DN patients and healthy controls was detected by RT-qPCR. Human renal epithelial cells HK-2 were incubated with D-glucose $(30 \mathrm{mmol} / \mathrm{l})$ to establish DN model in vitro. RTqPCR analysis confirmed the transfection effects and detected the expressions of TNF- $\alpha$, IL6 and IL-1 $\beta$. Western blotting analysis determined the protein expression of Anxa2, Bcl-2, Bax, cleaved caspase- 3 and caspase- 3 . The production of ROS was detected by DCF-DA method and production of inflammatory cytokines was verified by ELISA assay. CCK-8 assay and TUNEL assay were performed to determine cell viability and apoptosis, respectively. Dual-luciferase reporter assay was performed to confirm the relationship between miR-151-3p and hsa_circ_0003928 or Anxa2.

Results: Hsa_circ_0003928 and Anxa2 mRNA levels were increased, whereas miR-151-3p was decreased in both HG-induced HK-2 cells and patients with DN. Hsa_circ_0003928 knockdown could decrease cell viability loss and apoptosis, increase Bcl-2 expression, and decrease Bax and cleaved caspase-3 expression. Besides, hsa_circ_0003928 knockdown suppressed HG-induced overproduction of ROS, TNF- $\alpha$, IL- 6 and IL- $1 \beta$. However, the effects made by miR-151-3p inhibition were opposite to those made by hsa_circ_0003928 knockdown. Furthermore, the binding sites between miR-151-3p and hsa_circ_0003928 or Anxa2 were predicted and verified. Protein expression of Anxa2 was suppressed by hsa circ_0003928 knockdown, which was rescued by miR-151-3p inhibition.

Conclusion: These results demonstrated that hsa_circ_0003928 could act as a sponge of miR-151-3p and regulate HG-induced inflammation and apoptosis partly through regulating Anxa2.

Keywords: diabetic nephropathy, hsa_circ_0003928, miR-151-3p, Anxa2

\section{Introduction}

Diabetes mellitus is a common metabolic disorder that is associated with chronic complications such as retinopathy and peripheral neuropathy. It has been recognized that diabetes can cause chronic renal impairment and lead to diabetic nephropathy (DN). It is reported that approximately $30 \sim 40 \%$ of diabetes patients will 
develop into DN. ${ }^{1}$ Currently, DN has been a leading cause of end-stage renal disease (ESRD) throughout the world. ${ }^{2}$ Renal tubular cell injury is known as one of the main characteristics of DN and tubular cells are proved to be primary targets of $\mathrm{DN}^{3}$ As DN shows multi-stage pathological presentations, exploration into the mechanism underlying tubular injury under high glucose may be an efficient therapeutic target to block the progression of DN.

Circular RNAs (circRNAs), a class of endogenous noncoding RNA, are characterized by a stably closed-loop lacking the 5' cap and the 3' end of the poly (A) tail, thus obtaining a high degree of stability and sequence conservation. ${ }^{4,5}$ circRNAs have been found to play an important role in the regulation of multiple diseases, including cancerous tumors, cardiovascular diseases, and diabetes mellitus and its complications. ${ }^{6-9}$ For example, circRNA_15698 was validated to be upregulated in both $\mathrm{db} / \mathrm{db}$ mice and mouse mesangial cells exposed to high glucose, aggravating the extracellular matrix accumulation and deteriorating DN via miR-185/TGF- $\beta 1 .{ }^{9}$ This showed that circRNAs could participate in the progression of DN by acting as a miRNA sponge. RNA sequencing profiling from control and high glucose-induced human umbilical vein endothelial cells (HUVECs) identified 214 differentially expressed circRNAs, among which hsa_circ_0003928 was found to be upregulated in high glucose-induced HUVECs, ${ }^{10}$ indicating that hsa circ_0003928 might be involved in the pathogenesis of diabetes mellitus. However, whether hsa_circ_0003928 is correlated with DN is unknown. Besides, whether hsa_circ_0003928 can act as a miRNA sponge is also poorly understood. In this study, we have reported the expression and function of hsa_circ_0003928 and its regulatory mechanism in high glucose-induced HK-2 cells, providing a novel insight for DN pathogenesis.

\section{Patients and Methods}

\section{Patients and Samples}

We recruited participants who visited our center for a routine health check-up or treatment of diabetes mellitus at an outpatient clinic from January 2018 to December 2018. Finally, 60 participants, including 30 healthy volunteers and 30 patients with $\mathrm{DN}$, were enrolled. The diagnosis of DN was histologically confirmed after kidney biopsy by an experienced renal pathologist. The inclusion criteria were: 1) the indications for performing the renal biopsy were proteinuria with or without microscopic hematuria and fast drop in renal function; 2) type w diabetic patients with no history of using renal toxic or herbal medicine; 3) diabetes patients with no complications of other kidney diseases. Patients were excluded if they demanded any of following criteria: history of cardiovascular disease, morbid obesity, organic or inflammatory diseases, infectious, autoimmune, haematological, malignant, fever and diabetic neuropathy. All blood samples were obtained at least $8 \mathrm{~h}$ after fasting and collected at $-80^{\circ} \mathrm{C}$ for further analysis. This project was conducted according to the Helsinki Declaration and approved by the Ethics Committee of the Chongqing University Three Gorges Hospital. All participants provided their informed written consent.

\section{Cell Culture and Treatment}

The human tubular epithelial cell line HK-2 was obtained from the American Type Culture Collection (Manassas, VA, USA). Cells were cultured in DMEM/F12 culture medium (HyClone, USA) supplemented with 10\% FBS, $100 \mu / \mathrm{mL}$ penicillin and $100 \mathrm{mg} / \mathrm{mL}$ streptomycin in a $37^{\circ} \mathrm{C}$ humidified incubator containing a $5 \% \mathrm{CO}_{2}$ atmosphere. For the control group, the medium contained $5 \mathrm{mmol} / \mathrm{l} \mathrm{D}$-glucose; for experimental group, HK-2 cells were incubated with the medium containing $30 \mathrm{mmol} / \mathrm{l} \mathrm{D}$-glucose for $48 \mathrm{~h}$ to produce a high-glucose model. Besides, medium with mannitol (MA; $30 \mathrm{mmol} / \mathrm{l}$ ) was used as negative control.

\section{Cell Transfection}

HK-2 cells were transfected with siRNA, miR-151-3p mimic, miR-151-3p inhibitor and their corresponding negative control using Lipofectamine 2000 (Invitrogen, CA, USA) in accordance with the manufacturer's instructions. The siRNA targeting hsa_circ_0003928 (siRNA-circ_0003928-1 (GCCA ATTCCTTTGTGGGAA)) and (siRNA-circ_0003928-2 (GCAGAAATGGAGAAACTTA)) was used to knock down has_circ_0003928 and siRNA negative control (siRNA-NC) was used as a control. Besides, miR-151-3p mimic, mimic control, miR-151-3p inhibitor and inhibitor control were used to regulate the expression level of miR-151-3p. All the plasmids were provided by GenePharma (Shanghai, China). After transfection for $48 \mathrm{~h}$, HK-2 cells were collected for transfection efficacy detection by RT-qPCR.

\section{Cell Viability}

Cell viability was determined using a Cell Counting Kit-8 (CCK-8; Beyotime Institute of Biotechnology, Shanghai, 
China). HK-2 cells were inoculated into 96-well plates with $5 \times 10^{3}$ cell/well and treated with normal glucose $(\mathrm{NG} ; 5 \mathrm{mmol} / \mathrm{l})$ or high glucose $(\mathrm{HG} ; 5 \mathrm{mmol} / \mathrm{l})$ for 48 h. After incubation, $10 \mu \mathrm{L}$ of CCK-8 solution was added into each well for $1 \mathrm{~h}$ at $37^{\circ} \mathrm{C}$. The absorbance at $450 \mathrm{~nm}$ was recorded using a micro-plate reader.

\section{Cellular Apoptosis Assay}

Cellular apoptosis was determined by a terminal deoxynucleotidyl transferase dUTP nick end labeling (TUNEL) assay (Millipore; Merck KGaA, Darmstadt, Germany). HK-2 cells were seeded on chamber slides at a density of $4 \times 10^{3}$ cell/well. After incubation for $48 \mathrm{~h}, \mathrm{HK}-2$ cells were fixed in $4 \%$ paraformaldehyde, permeabilized with $0.1 \%$ Triton X-100 in PBS, and incubated with TUNEL reagents. The apoptotic cells were observed under an optical microscope (Olympus Corp., Tokyo, Japan).

\section{Reverse Transcription-Quantitative Polymerase Chain Reaction (RT-qPCR)}

Total RNA from HK-2 cells was extracted using TRIzol reagent (Life Technologies Corporation, Carlsbad, CA, USA) and reversely transcribed to complementary DNA using Prime Script RT kit (Takara Bio, Inc., Otsu, Japan) in accordance with the manufacturer's instructions. Then, the relative mRNA expression was quantified using an ABI 7500 Real-time PCR system (Applied Biosystems; Thermo Fisher Scientific, Inc., Rockford, IL, USA) with the SYBR Green kit (Takara Bio, Inc.). The primers were used as follows: hsa_circ_0003928, forward: 5'- CCTAG AGCTGGAATGCCGTC-3', reverse: 5'- ATGCTGTCGG AGTAGCATGG-3'; TNF- $\alpha$, forward: 5'-CCCAGGGA CCTCTCTCTAATC-3', reverse: 5'- GTTTGCTACAAC ATGGGCTACAG-3'; IL-1 $\beta$, forward: 5'- TGGTGAT GTCTGGTCCATATGAAC-3', reverse: 5'- CCAA GGCCACAGGTATTTTGTC-3'; IL-6, forward: 5'- GGTA CATCCTCGACGGCATCT-3', reverse: 5'-GTGC CTCTTTGCTGCTTTCAC-3'; miR-151-3p, forward: 5'GGATGCTAGACTGAAGCTCCT-3', reverse: 5'- CAG TGCGTGTCGTGGAGT-3'; Anxa2, forward: 5'- CACG GCCCAGGTTATCTTGT-3', reverse: 5'-TGCTGCGG TTGGTCAAAATG-3' $\beta$-actin, forward: 5'-CGTG AAAAGATGACCCAGATCA-3', reverse: 5'- CACAG CCTGGATGGCTACGT-3'; U6, forward: 5'-CTCGCTT CGGCAGCACA-3', reverse: 5'-AACGCTTCACGAA
TTTGCGT-3'. $\beta$-actin and U6 were used as internal references for normalizing gene expression.

\section{Western Blotting}

Total protein from HK-2 cells was extracted using RIPA lysis buffer (Thermo Fisher Scientific, Inc.) supplemented with $1 \mathrm{mM}$ phenylmethylsulfonyl fluoride (PMSF) and protease inhibitor cocktail (Roche, Basel, Switzerland), and the protein concentration was determined using a BCA protein assay kit (Thermo Fisher Scientific, Inc.). Equal amount of protein $(40 \mu \mathrm{g})$ of each sample was separated with SDS-PAGE and transferred onto PVDF membranes. The membranes were blocked in TBST buffer containing $5 \%$ skimmed milk for $1 \mathrm{~h}$ at room temperature and subsequently incubated with primary antibodies against Bcl-2 (1:1000; cat. no. ab196495), Bax (1:1000; cat. no. ab182733), Cleaved caspase-3 (1:500; cat. no. ab2302), caspase-3 (1:500; cat. no. ab13847), Anxa2 (1:1000; cat. no. ab41803) and GAPDH (1:1000; cat. no. ab181603; all purchased from Abcam), followed by incubation with horseradish peroxidase-labeled anti-rabbit secondary antibody (1:2000; cat. no. sc-2004; Santa Cruz Biotechnology, Inc.) at an appropriate dilution for $1 \mathrm{~h}$ at room temperature. The protein bands were developed using an enhanced chemiluminescence reagent system (Thermo Fisher Scientific, Inc.). GAPDH was used as the loading control.

\section{Enzyme-Linked Immunosorbent Assay}

The production of TNF- $\alpha$, IL- 6 and IL- $1 \beta$ in the supernatant of cells in each group was measured using Enzymelinked immunosorbent assay (ELISA). The corresponding ELISA kits (BD Bioscience, USA) for TNF- $\alpha$, IL- 6 and IL- $1 \beta$ detection were used in accordance with the manufacturer's instructions.

\section{Reactive Oxygen Species (ROS) Production}

The intracellular ROS production was measured using 2', 7'-dichlorodihydrofluorescein diacetate (DCF-DA; Sigma Aldrich). Cells were incubated in a 6-well plate for $48 \mathrm{~h}$. After incubation with $5 \mu \mathrm{M}$ DCF-DA for $1 \mathrm{~h}$, HK-2 cells were harvested and washed with PBS, and the fluorescence intensity was detected at the wavelength 488/525 $\mathrm{nm}$ and analyzed using a BD FACSCalibur flow cytometer. 


\section{Luciferase Reporter Assay}

We obtained reporter plasmids by inserting hsa_circ_0003928 or ANXA2 3'UTR sequence containing wild-type (WT) or mutant (MUT) miR-151-3p-binding site into the pmirGLO vector (Promega, Madison, WI, USA). HEK293 cells were co-transfected with reporter plasmids and miR-151-3p mimic or mimic control using Lipofectamine 2000. Forty-eight hours after transfection, the luciferase activities were tested by the Dual-Luciferase Reporter Assay system (Promega).

\section{Statistical Analysis}

All experiments were repeated at least three times. All data were presented as mean \pm standard deviation (SD). Statistical analysis was carried out using SPSS 19.0 statistical software (SPSS Inc., Chicago, IL). Student's $t$-test and ANOVA followed by post hoc Tukey's test were exploited to analyze significance difference. A value of $\mathrm{P}<0.05$ was considered significant.

\section{Results}

\section{Hsa_circ_0003928 Was Upregulated in} Serum of DN Patients and Cell Model in vitro

To detect hsa_circ_0003928 expression upon D-glucose stimulation, HK-2 cells were exposed to different concentrations of D-glucose $(5,10,15,20,25$ and $30 \mathrm{mmol} / \mathrm{l})$, regarding mannitol (MA) as the negative control. We found that the expression level of hsa_circ_0003928 was upregulated upon D-glucose stimulation with a concentration-dependent manner, and hsa_circ_0003928 expression was not affected by MA (Figure 1A). Subsequent experiments exhibited an upregulation of hsa_circ_0003928 expression with the increasing time for D-glucose ( $30 \mathrm{mmol} / \mathrm{l})$ incubation (Figure 1B). In addition, compared to the healthy volunteers, the expression level of hsa_circ_0003928 was significantly increased in serum of patients with DN (Figure 1C).

\section{Hsa_circ_0003928 Knockdown Suppressed Cell Viability Loss and Apoptosis in HG-Induced HK-2 Cells}

To further investigate the role of hsa_circ_0003928 in HGinduced HK-2 cells, siRNA-circ_0003928-1/2 was used for transfection to knock down hsa_circ_0003928, and siRNA-circ_0003928-1, which showed a higher transfection efficacy than siRNA-circ_0003928-2, was used for the next experiments (Figure 2A). CCK-8 assay showed that cell viability was significantly decreased upon exposure to HG, but it was reversely increased by hsa_circ_0003928 knockdown (Figure 2B). HG resulted in an obvious morphology of cell apoptosis by TUNEL staining, as the cells exhibited obvious nuclear shrinkage and the nuclear was stained brown, which was then alleviated by hsa_circ_0003928 knockdown (Figure 2C). In addition, apoptosis-related proteins were further detected. As shown in Figure 2D and E, HG remarkably induced a decrease of protein expression of Bcl-2 and the increases of protein expression of Bax and cleaved caspase-3, demonstrating a remarkably high cell apoptotic rate induced by $\mathrm{HG}$ stimulation. However, cell apoptosis was attenuated by hsa_circ_0003928 knockdown as the protein expression of Bcl-2 was increased and the protein expressions of Bax and cleaved caspase- 3 were decreased upon hsa_circ_0003928 knockdown.
A

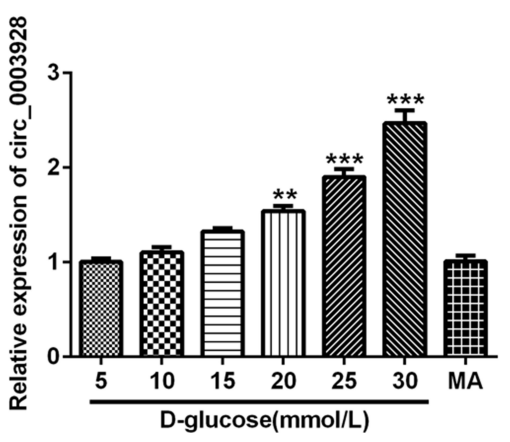

B

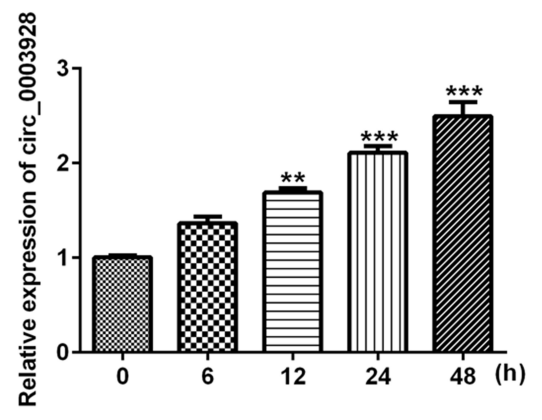

C

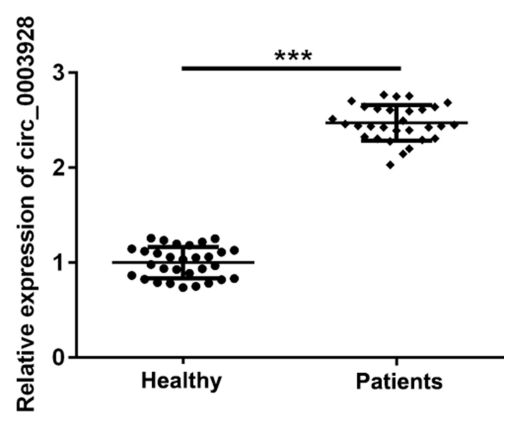

Figure I Hsa_circ_0003928 was upregulated in high D-glucose-induced HK-2 cells and serum of patients with DN. (A) HK-2 cells were subjected to different concentrations of D-glucose $(5,10,15,20,25$ and $30 \mathrm{mmol} / \mathrm{l})$ for $48 \mathrm{~h}$. Besides, mannitol (MA; 30 mmol/l) was used as negative control. The expression level of hsa_circ_0003928 was measured by RT-qPCR. **, ***p<0.0I, 0.001 vs $5 \mathrm{mmol} / \mathrm{l}$ D-glucose. (B) HK-2 cells were subjected to $30 \mathrm{mmol} / \mathrm{l} \mathrm{D-glucose}$ for $6,12,24$ and 48 h, respectively. The expression level of hsa_circ_0003928 was measured by RT-qPCR. **, ***p<0.0I, $0.00 \mathrm{I}$ vs $0 \mathrm{~h}$. (C) The expression level of hsa_circ_0003928 in serum of healthy control and patients with DN was measured by RT-qPCR. ***p<0.00I.

Abbreviations: DN, diabetic nephropathy; MA, mannitol. 
A

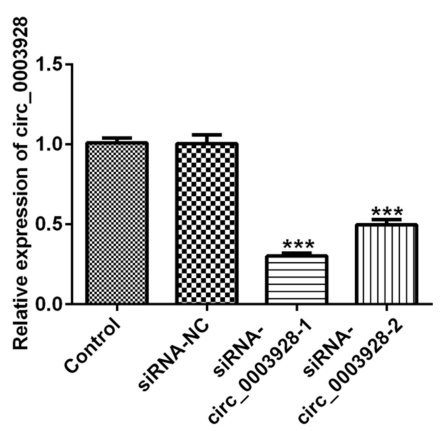

B

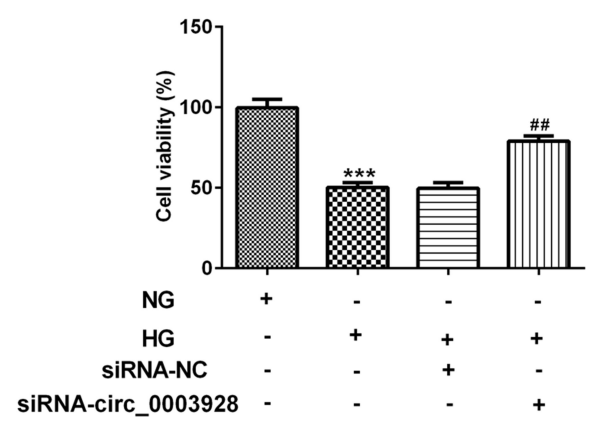

C

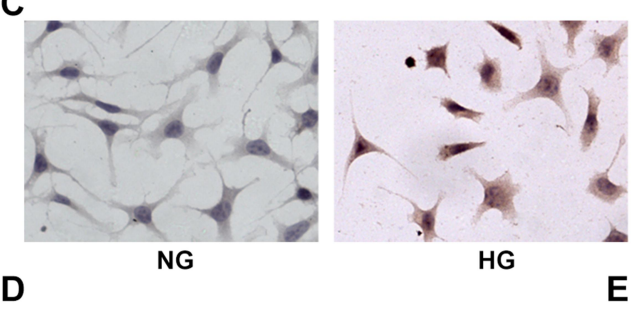

$\mathbf{E}$
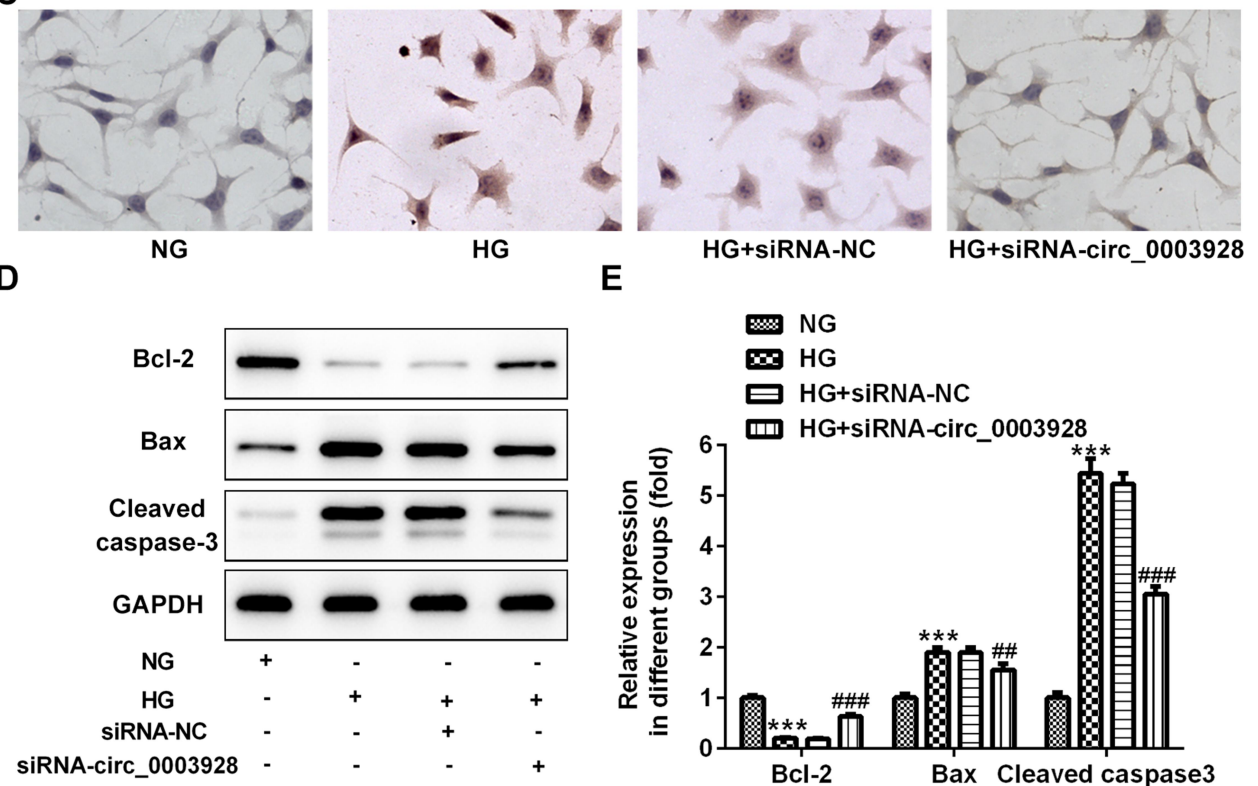

Figure 2 Hsa circ 0003928 knockdown suppressed cell viability loss and apoptosis in HG-induced HK-2 cells. (A) HK-2 cells were transfected with siRNA-circ 0003928$\mathrm{I} / 2$ and the siRNA negative control (siRNA-NC) using Lipofectamine 2000 , and the transfection efficacy was determined using RT-qPCR. *** $\mathrm{p}<0.00 \mathrm{I}$ vs siRNA-NC. (B) HK2 cells without transfection were treated with normal D-glucose (NG; 5 mmol/l) or high D-glucose (HG; 30 mmol/l) for 48 h. Besides, transfected HK-2 cells were inoculated into 96-well plates and treated with HG for 48 h. Then, CCK-8 assay was performed to determine cell viability of different groups. (C) TUNEL assay was conducted to determine HK-2 cell apoptosis. (D and E) The protein expression of Bcl-2, Bax, cleaved caspase-3 and GAPDH were detected using Western blotting, and the band intensities were quantified. GAPDH acted as the loading control, and the band intensity was normalized to GAPDH. $* * *$ p $<0.00 \mathrm{I}$ vs normal D-glucose (NG); ${ }^{\#,}$ \#\# $<0.01,0.001$ vs HG+siRNA-NC.

Abbreviations: HG, high D-glucose; NG, D-glucose; siRNA-circ_0003928, siRNA targeting hsa_circ_0003928; siRNA-NC, siRNA negative control.

\section{Hsa_circ_0003928 Knockdown}

\section{Suppressed Production of ROS and}

\section{Inflammatory Cytokines in HG-Induced} HK-2 Cells

We next investigated the effect of hsa_circ_0003928 on HG-induced ROS level and inflammation in HK-2 cells. First, ROS level in each group was detected by DCF-DA assay, and the results revealed that hsa_circ_0003928 knockdown could inhibit ROS level which was elevated by HG stimulation (Figure 3A). Then, ELISA and RTqPCR were conducted, respectively, to detect the concentrations and mRNA levels of inflammatory cytokines. As shown in Figure 3B-D, the concentrations of TNF- $\alpha$, IL-6 and IL-1 $\beta$ in culture supernatant were significantly upregulated in HG group, which were then downregulated after hsa_circ_0003928 was knocked down in HG-induced HK2 cells. In addition, total RNA was extracted from HK-2 cells in different groups, and the mRNA levels of TNF- $\alpha$, IL- 6 and IL- $1 \beta$ exhibited similar changes with the ELISA results (Figure $3 \mathrm{E}-\mathrm{G}$ ).

\section{Hsa_circ_0003928 Binds with miR-I5I- $3 p$}

According to the bioinformatics assay (https://circinter actome.nia.nih.gov), hsa_circ_0003928 was predicted to directly target miR-151-3p. This prediction was then 


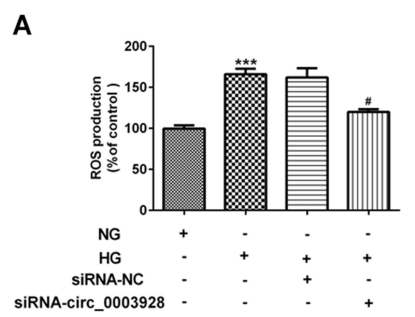

E

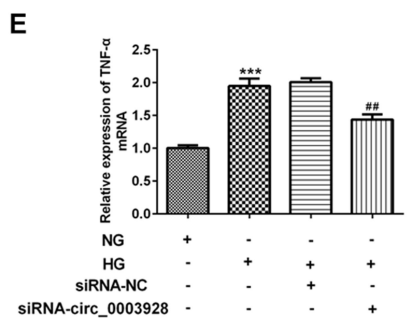

B

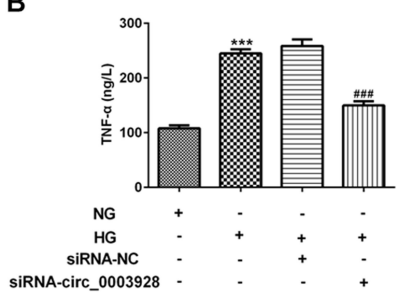

F
C

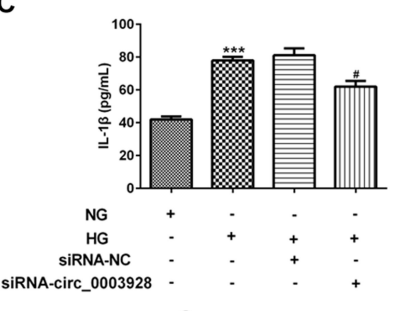

D

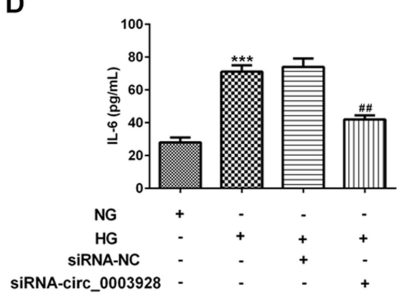

G
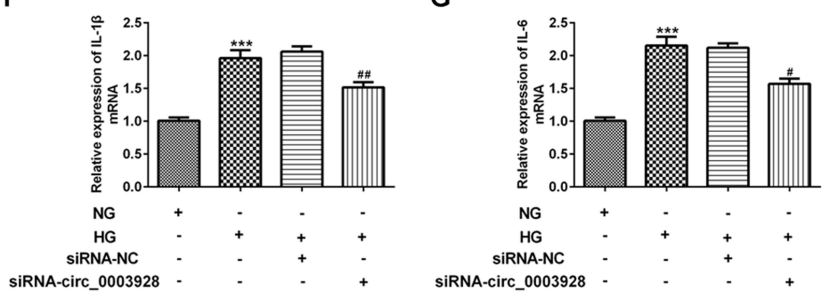

Figure 3 Hsa_circ_0003928 knockdown suppressed production of ROS and inflammatory cytokines in HG-induced HK-2 cells. (A) HK-2 cells without transfection were treated with NG or HG for 48 h. Besides, HK-2 cells transfected with siRNA-circ_0003928 or siRNA-NC were inoculated into $96-$-well plates and treated with HG for 48 h. Production of ROS was detected by DCF-DA method. (B-D) The production of TNF- $\alpha$, IL- 6 and IL-I $\beta$ was determined using corresponding ELISA kits. (E-G) The mRNA levels of TNF- $\alpha$, IL-6 and IL-I $\beta$ were detected using RT-qPCR. $* * * p<0.001$ vs NG; \#, \#, \#\# $p<0.05,0.01,0.001$ vs HG+siRNA-NC.

Abbreviations: HG, high D-glucose; NG, D-glucose; siRNA-circ_0003928, siRNA targeting hsa_circ_0003928; siRNA-NC, siRNA negative control; ROS, reactive oxygen species; DCF-DA, 2', 7'-dichlorodihydrofluorescein diacetate.

verified by luciferase reporter assay, as co-transfection of miR-151-3p mimic and wild-type (WT) hsa_circ_0003928 weakened the luciferase activity of the WT hsa_circ_0003928 reporter (Figure 4A and B). These results suggested that hsa_circ_0003928 might be a direct functional target of miR-151-3p. As shown in Figure 4C, hsa_circ_0003928 knockdown significantly improved the expression level of miR-151-3p. Besides, miR-151-3p was found to be significantly downregulated in HG-induced HK-2 cells and serum of patients with DN (Figure 4D and E).

\section{Anxa2 is a Direct Target of miR-I5I-3p}

StarBase software (http://starbase.sysu.edu.cn) predicted a binding site between miR-151-3p and Annexin A2 (Anxa2), and the subsequent luciferase reporter assay demonstrated the relationship that Anxa2 might be a direct target of miR-151-3p (Figure 5A and B). Next, RT-qPCR and Western blotting assays showed that miR151-3p overexpression significantly increased the expression of Anxa2 at mRNA and protein levels (Figure 5C-E). In addition, both the mRNA level and protein expression of Anxa2 was found to be upregulated in HG-induced HK2 cells (Figure 5F-H). Accordingly, the mRNA level of Anxa2 was also upregulated in serum of patients with DN (Figure 5F-I).

\section{Hsa_circ_0003928/miR-I5I-3p Axis Regulates HG-Induced HK-2 Cells via Anxa2}

Finally, we explored whether miR-151-3p inhibition could partly reverse the effects induced by hsa_circ_0003928 knockdown in HG-induced HK-2 cells via Anxa2. First, protein expression of Anxa2 was significantly increased when miR151-3p was inhibited, while was significantly decreased when hsa_circ_0003928 was silenced (Figure 6A and B), indicating that Anxa2 was regulated by hsa_circ_0003928/miR-151-3p axis. Then, a loss-of-function approach was used to conduct the following experiments. We found that miR-151-3p inhibition strengthened cell viability loss and apoptosis, as well as the downregulated protein expression of Bcl-2 and upregulated protein expression of Bax and cleaved caspase-3 in HK-2 cells upon $\mathrm{HG}$ exposure, whereas this function was effectively reversed by hsa_circ_0003928 knockdown (Figure 6C-E). In addition, overproduction of ROS, TNF- $\alpha$, IL- 6 and IL-1 $\beta$ induced by HG was strengthened by miR-151-3p inhibition but weakened by hsa_circ_0003928 knockdown. However, these effects were effectively reversed when miR-151-3p inhibitor and siRNA-circ_0003928 were applied simultaneously (Figure 7A-D). Thus, HG-induced viability loss, apoptosis and inflammation were regulated by hsa_circ_0003928/miR151-3p axis. Taking the regulatory effect of hsacirc_0003928/miR-151-3p axis on Anxa2 into consideration, 
A

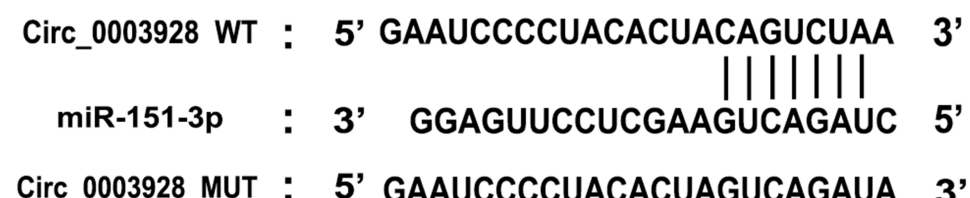

B

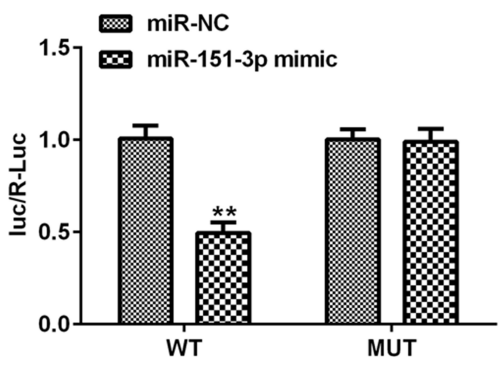

D

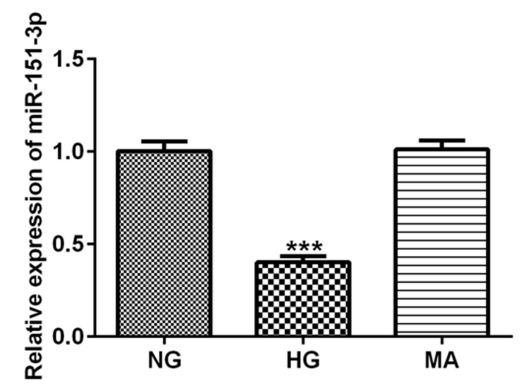

C

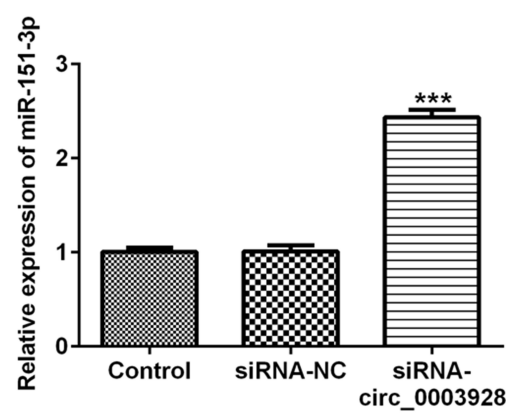

$\mathbf{E}$

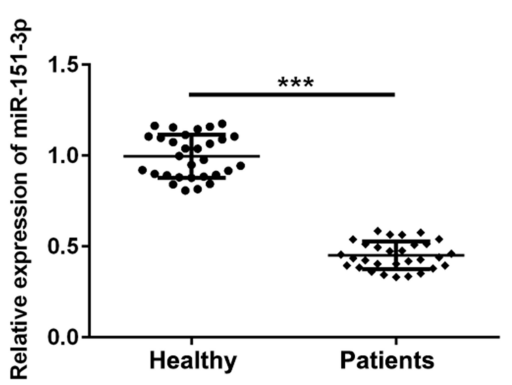

Figure 4 Hsa_circ_0003928 binds to miR-I5I-3p. (A) According to the bioinformatics assay (https://circinteractome.nia.nih.gov), a binding site between hsa_circ_0003928 and miR-I5I-3p was predicted. (B) HEK293 cells were co-transfected with wild-type (WT) hsa_circ_0003928 or mature (MUT) hsa_circ_0003928 and miR-I5I-3p mimic or miR-NC (mimic control) using Lipofectamine 2000. $48 \mathrm{~h}$ after transfection, the luciferase activities were tested by the Dual-Luciferase Reporter Assay system. ** $<<0.0 \mathrm{I}$ vs miR-NC. (C) HK-2 cells were transfected with siRNA-NC or siRNA-circ_0003928, and the expression level of miR-I5I-3p was detected by RT-qPCR. *** $<0.001$ vs siRNA-NC. (D) HK-2 cells were treated with NG, HG and MA, respectively, and the expression level of miR-I5I-3p was detected by RT-qPCR. **** $<0.00$ I vs NG. (E) The expression level of miR-15I-3p in serum from healthy control and patients with DN was measured by RT-qPCR. *** $\mathrm{p}<0.001$.

Abbreviations: HG, high D-glucose; NG, D-glucose; siRNA-circ_0003928, siRNA targeting hsa_circ_0003928; siRNA-NC, siRNA negative control; MA, mannitol; miRNC, mimic control; DN, diabetic nephropathy; WT, wild-type; MUT, mature.

our results indicated that hsa_circ_0003928 knockdown attenuated HG-induced cell injuries targeting miR-151-3p partly via Anxa2.

\section{Discussion}

Diabetes mellitus with the escalating incidence often engenders the occurrence of microangiopathic complications including DN. Currently, DN has developed in more than one-third of patients with diabetes mellitus and has become the leading cause of ESRD, posing a serious threat to the governments and researchers. ${ }^{11,12}$ Renal tubular damage can be evidently found in patients with $\mathrm{DN}$, and a large amount of researchers have suggested that renal tubular epithelial cells death is involved in DN. ${ }^{13,14}$ Importantly, inflammatory response and apoptosis of renal tubular cells are found in both DN patients and DN animal models, mainly accounting for the pathogenesis of tubular lesion in DN. ${ }^{15,16}$ Many factors account for renal injury by controlling inflammatory cytokine production and cell apoptosis, including IL-17a, miR-34b and TRPC6. ${ }^{17-19}$ Therefore, the use of effective therapeutic agents that specifically suppress inflammatory injury and apoptosis may be useful strategies for renal function protection.

Here, human renal tubular epithelial HK-2 cells were stimulated by $\mathrm{HG}$ to simulate $\mathrm{DN}$ in vitro, accompanied by severe cell viability loss, ROS overproduction, inflammatory response and cell apoptosis. Besides, we found that hsa circ_0003928 and Anxa2 were increased, whereas miR-151$3 p$ was decreased in both HG-induced HK-2 cells and serum from patients with DN. There existed a regulatory relationship between hsa_circ_0003928 and miR-151-3p, as well as between miR-151-3p and Anxa2. A series of loss-offunctional experiments revealed that hsa_circ_0003928 knockdown could abolish HG-induced viability loss, inflammation, and apoptosis by downregulating miR-151-3p, while miR-151-3p inhibition strengthened HG-induced cell injuries. 
A

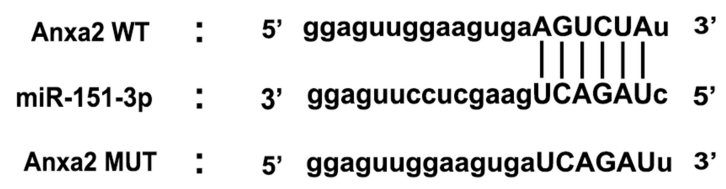

B

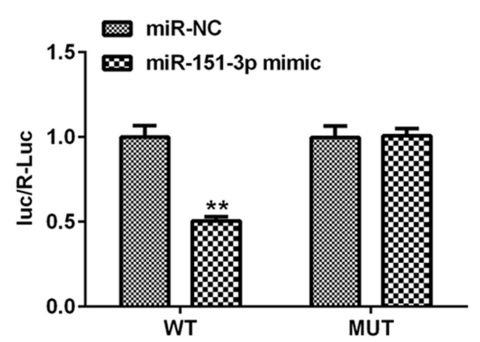

E

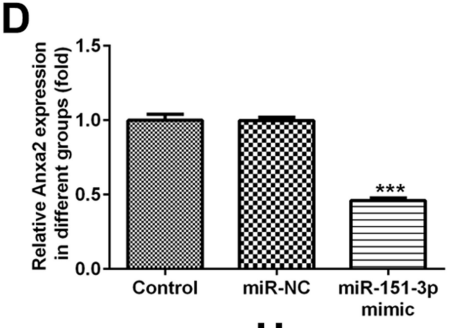

E

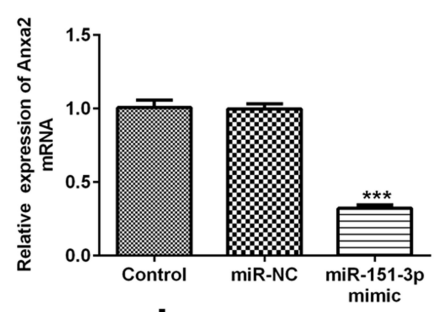

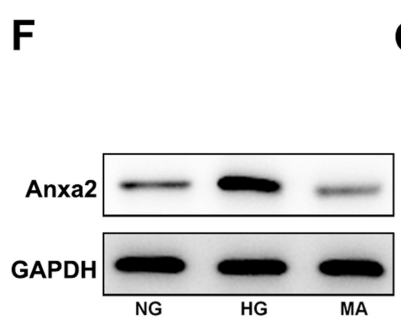
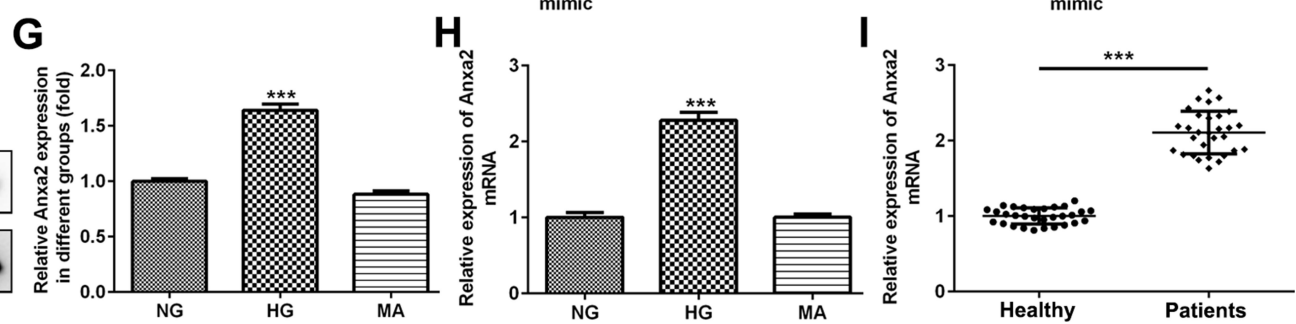

Figure 5 Anxa2 is a direct target of miR-15I-3p. (A) StarBase software (http://starbase.sysu.edu.cn) predicted a binding site between miR-I5I-3p and Annexin A2 (Anxa2). (B) HEK293 cells were co-transfected with wild-type (WT) Anxa2 or mature (MUT) Anxa2 and miR-I5I-3p mimic or miR-NC (mimic control) using Lipofectamine 2000. 48 $\mathrm{h}$ after transfection, the luciferase activities were tested by the Dual-Luciferase Reporter Assay system. $* *$ p $<0.0$ I vs miR-NC. (C-E) HK-2 cells were transfected with miRI5I-3p mimic and miR-NC, then the protein expression and mRNA level of Anxa2 were detected using Western blotting and RT-qPCR, respectively. *** $<<0.00 \mathrm{I}$ vs miR-NC. (F-H) HK-2 cells were treated with NG, HG and MA, respectively, and the protein expression and mRNA level of Anxa2 were detected using Western blotting and RTqPCR, respectively. GAPDH acted as the loading control, and the band intensity of Anxa2 was normalized to GAPDH. ***p<0.00I vs NG. (I) The mRNA level of Anxa2 in serum from healthy control and patients with DN was measured by RT-qPCR. *** $p<0.001$.

Abbreviations: HG, high D-glucose; NG, D-glucose; MA, mannitol; miR-NC, mimic control; DN, diabetic nephropathy; WT, wild-type; MUT, mature.

Ultimately, these results demonstrated that hsa_circ_0003928 could act as a sponge of miR-151-3p and regulate HG-induced inflammation and apoptosis partly through regulating Anxa2 (Figure 8).

As promising as these results have been, studies focusing on the specific mechanism of circRNAs function in the pathological process of DN are far from adequate. To date, the most canonical regulatory mode of circRNAs is miRNA sponge. ${ }^{20}$ This theory has been adequately elaborated for circRNAs by competitively harboring miRNAs. Recent emerging evidence indicated that some circRNAs exhibited aberrant expression in DN and regulate the development of DN. For example, circ_0080425 was upregulated in mouse DN model and high glucose-induced mesangial cells, and circ_0080425 promoted mesangial cell proliferation and fibrosis via competitively binding to miR-24-3p, which subsequently promoted DN progression. ${ }^{21}$ circ_0000285 promoted podocyte injuries via sponging miR-654-3p and activating MAPK6 in $\mathrm{DN}^{22}$ In this study, we identified that hsa_circ_0003928 acted as a sponge for miR-151-3p. MiR-151-3p is mapped to a region of chromosome $8 \mathrm{q}$, and an abnormally amplified miR-151-3p has been found in cancers, chronic hepatitis $\mathrm{B}$, and diabetes. ${ }^{23-25}$ A previous study reported that miR-151-3p level was decreased at late-stage diabetes in Zucker diabetic rats. Besides, miR-151-3p could function as an important regulator in the immune response and inflammation and inhibit LPS-induced IL-6 production. ${ }^{26}$ However, to our knowledge, the role of miR-151-3p in DN remains unclear and we are the first to report its role in DN. Here, miR-151-3p was demonstrated to be downregulated in HG-induced HK-2 cells and patients with DN, in line with its downregulation in diabetes. Inhibition of miR-151-3p promoted HK-2 cell injuries and weakened the protective effects of hsa_circ_0003928.

Anxa2, a $36-\mathrm{kDa}$ protein belonging to the calciumdependent phospholipid-binding proteins, is one of the molecules required to maintain the antithrombogenic properties of endothelial cells. ${ }^{27}$ Anxa2 has been demonstrated to be upregulated in glomeruli in diabetic KKAy mice, and the 
A

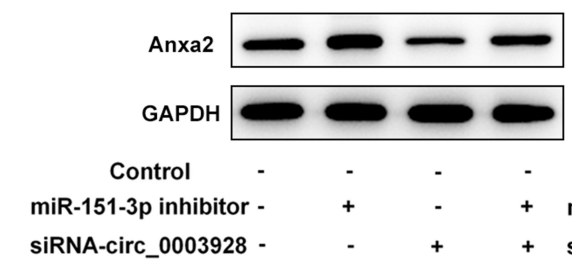

D

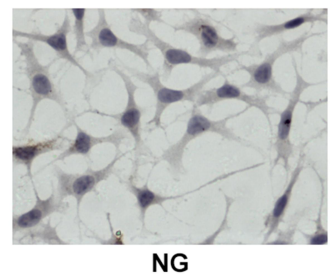

E

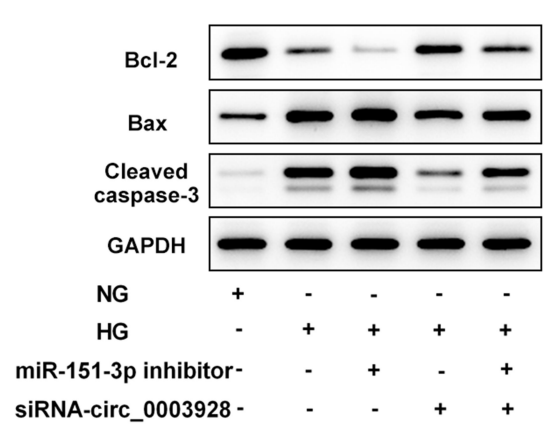

B

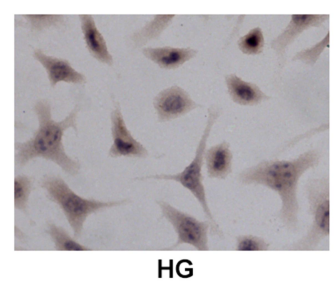

HG

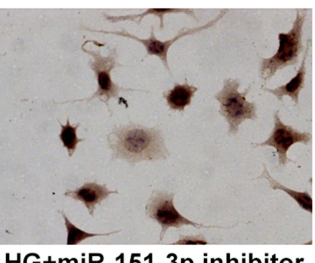

HG+miR-151-3p inhibitor

Control -

iR-151-3p inhibitor -

siRNA-circ_0003928 -

C

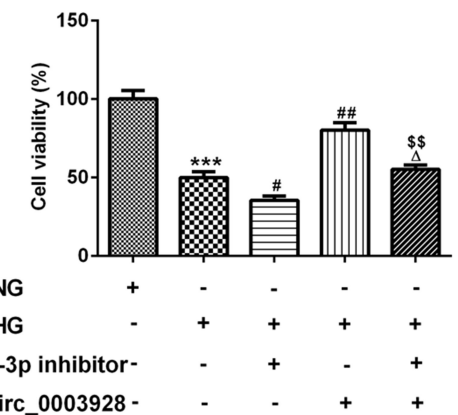

siRNA-circ_0003928 -
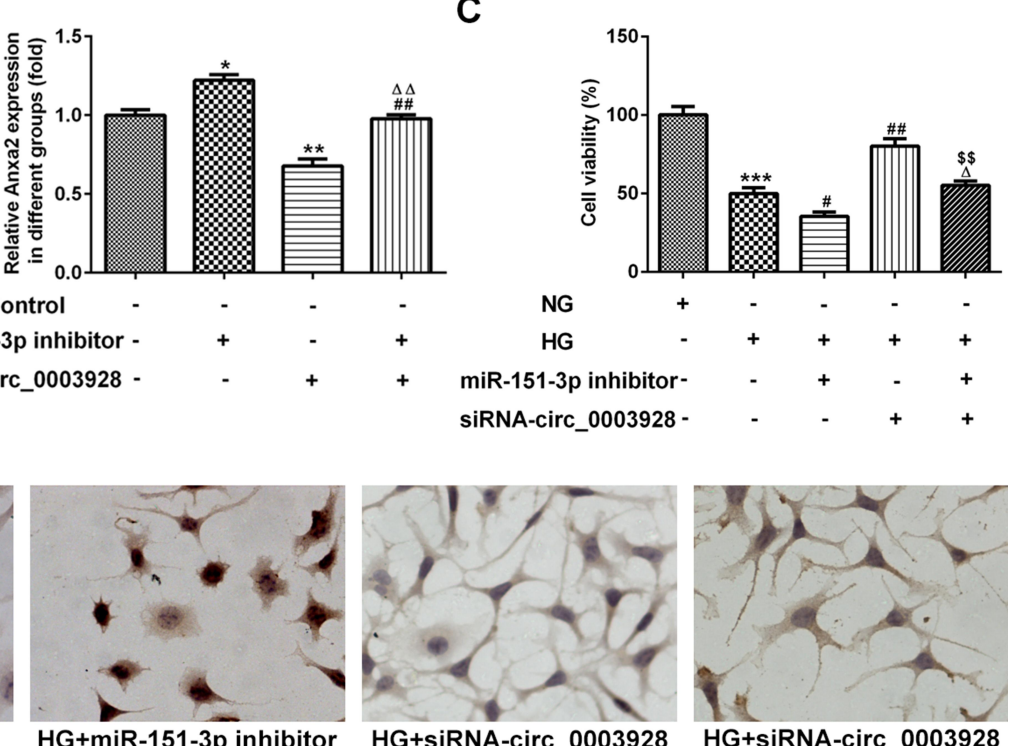

HG+siRNA-circ_0003928 +miR-151-3p inhibitor

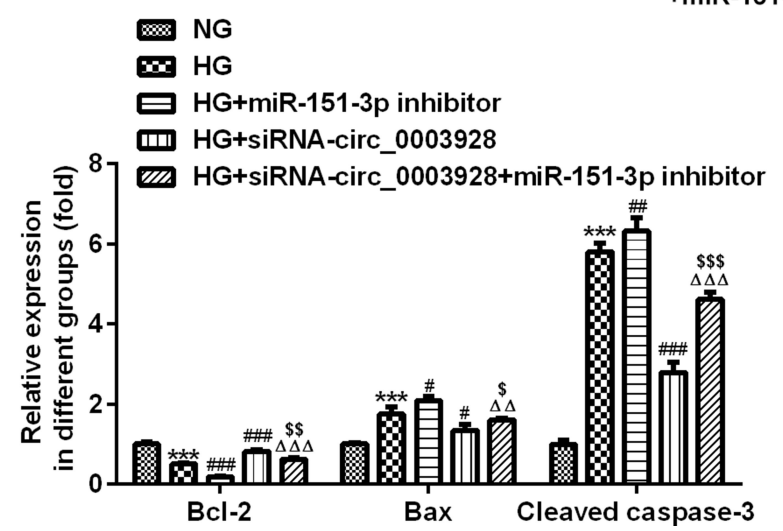

Figure 6 Hsa_circ_0003928/miR-I5I-3p axis regulates HG-induced viability loss and apoptosis in HK-2 cells. (A and B) HK-2 cells were transfected with miR-I5I-3p inhibitor or siRNA-circ_0003928, or co-transfected with miR-I5I-3p inhibitor and siRNA-circ_0003928, then the protein expression of Anxa2 was detected using Western blotting. *, **p<0.05, 0.01 vs control; \# $\#<0.01$ vs miR-I5I-3p inhibitor; $\Delta \Delta \mathrm{p}<0.01$ vs siRNA-circ_0003928. (C) HK-2 cells were treated with NG or HG for 48 h. Besides, after transfection with miR-15I-3p inhibitor or siRNA-circ_0003928 or the combination of iR-15I-3p inhibitor and siRNA-circ_0003928, HK-2 cells were treated with HG for $48 \mathrm{~h}$, then cell viability of different groups was assayed using CCK-8. (D) TUNEL assay was conducted to determine HK-2 cell apoptosis. (E) The protein expression of $\mathrm{Bcl}-2$, Bax, cleaved caspase-3, and GAPDH were detected using Western blotting, and the band intensities were quantified. GAPDH acted as the loading control, and the

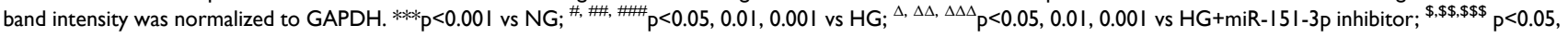
$0.01,0.001$ vs HG+siRNA-circ_0003928.

Abbreviations: HG, high D-glucose; NG, D-glucose; siRNA-circ_0003928, siRNA targeting hsa_circ_0003928.

upregulated Anxa2 participated in the mechanism relative to vitamin D insufficiency during early-stage DN, accelerating the development of DN. ${ }^{28}$ Specific expression network analysis of diabetic nephropathy kidney tissue also suggested that Anxa2 was a key gene during the development of DN. ${ }^{29}$ Furthermore, Anxa2 gene silencing was able to alleviate obesity-induced insulin resistance via suppressing the nuclear factor kappa B (NF-kB) signaling pathway. ${ }^{30}$ Therefore, Anxa2 possibly exerts its function in inflammatory environment by regulating NF- $\kappa B$. Moreover, the function of Anxa2 on cell apoptosis also received attention in recent years. For example, Anxa2 was highly expressed in acute pancreatitis, accompanied with a relatively high apoptotic rate. Anxa2 overexpression induced proapoptotic effects on rat pancreatic acinar cell, while Anxa2 knockdown exhibited the opposite effects. ${ }^{31}$ In this study, an upregulation of Anxa2 was discovered in serum of patients with DN and HG-induced HK-2 cells. In addition, Anxa2 was demonstrated to be a target of miR-151-3p. Hsa_circ_0003928 knockdown repressed Anxa2 protein expression, which was reversed by miR-151-3p inhibition in vitro. In addition, considering the important role of Anxa2 in regulating inflammation and apoptosis, the regulatory effect of hsa_circ_0003928/miR-151-3p in HG-induced cell viability loss, apoptosis and inflammation in HK-2 cells was possibly dependent on Anxia regulation. To sum 
A

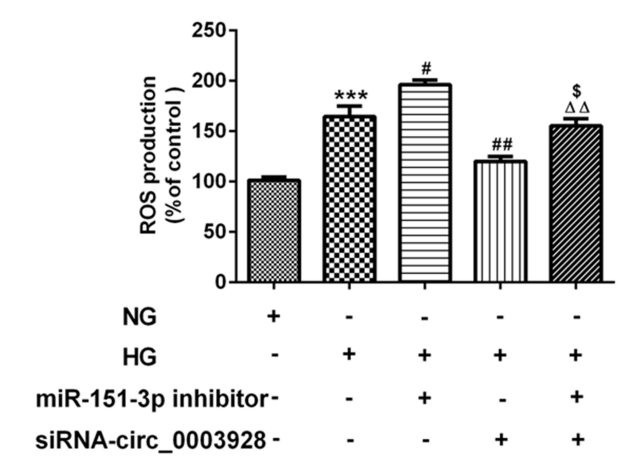

C

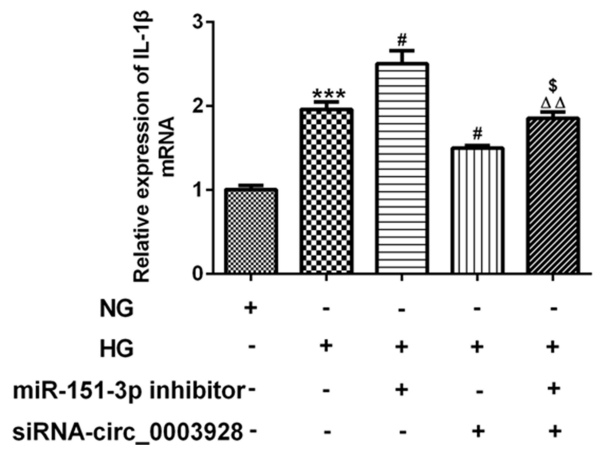

B

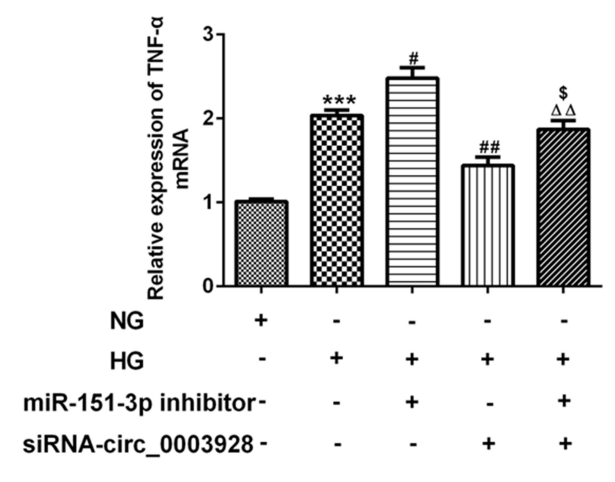

D

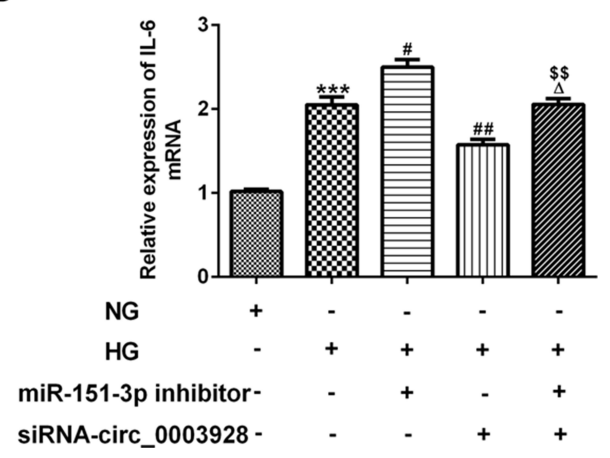

Figure 7 Hsa_circ_0003928/miR-I5I-3p axis regulates HG-induced inflammatory response in HK-2 cells. (A) HK-2 cells were treated with NG or HG for 48 h. Besides, after transfection with miR-I5I-3p inhibitor or siRNA-circ_0003928 or the combination of iR-I5I-3p inhibitor and siRNA-circ_0003928, HK-2 cells were treated with HG for $48 \mathrm{~h}$, then production of ROS was determined using DCF-DA method. (B-D) The mRNA levels of TNF- $\alpha$, IL-6 and IL-I $\beta$ were determined by RT-qPCR. *** $\mathrm{P}<0.00 \mathrm{I}$ vs NG; ${ }^{\# \#}$ p $<0.05,0.01$ vs HG; ${ }^{\Delta, \Delta \Delta}$ p $<0.05,0.01$ vs HG+miR-15I-3p inhibitor; ${ }^{\$,} \$ \$ p<0.05,0.01$ vs HG+siRNA-circ_0003928.

Abbreviations: HG, high D-glucose; ROS, reactive oxygen species; siRNA-circ_0003928, siRNA targeting hsa_circ_0003928; DCF-DA, 2', 7'-dichlorodihydrofluorescein diacetate.

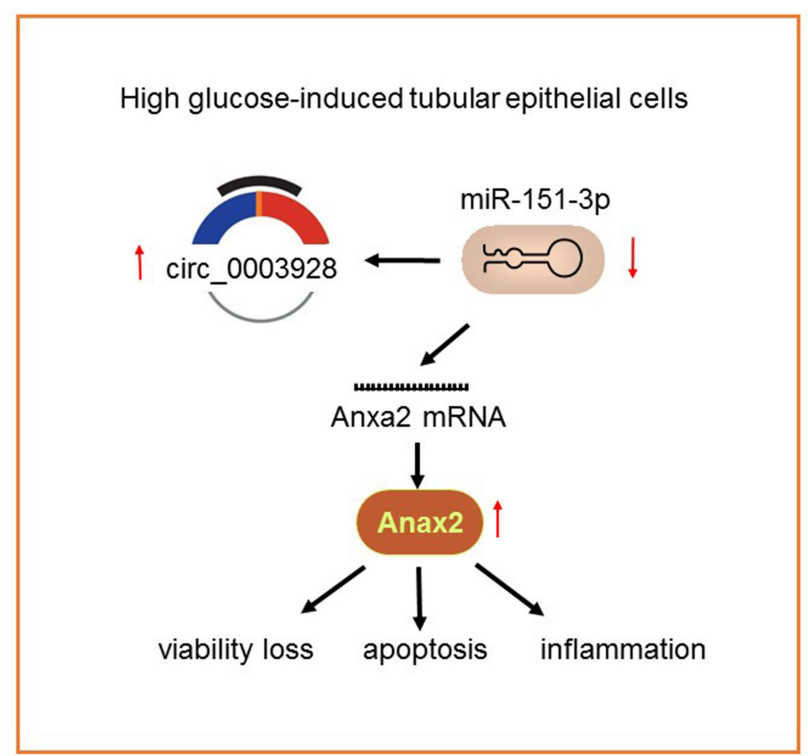

Figure 8 An illustration showing that high-glucose induced overexpression of has_circ_0003928 promoted cell viability loss, apoptosis and inflammation in HK2 cells, possibly via regulating miR-I5I-3p/Anxa axis. up, a novel mechanism of hsa_circ_0003928/miR-151-3p/ Anxa2 in DN progression was indicated in our study.

\section{Conclusion}

Taken together, all these data above have verified that hsa_circ_0003928 knockdown can withstand HGinduced cell injuries via suppressing inflammation and cell apoptosis. Also, hsa_circ_0003928 can directly bind to miR-151-3p and regulate Anxa2 expression. Hence, we conjecture that hsa_circ_0003928 knockdown might attenuate HG-induced cell injuries by targeting miR151-3p partly via Anxa2, providing a novel and promising target for $\mathrm{DN}$.

\section{Disclosure}

The authors declare that they have no potential conflicts of interest for this work. 


\section{References}

1. Helou N, Dwyer A, Shaha M, Zanchi A. Multidisciplinary management of diabetic kidney disease: a systematic review and meta-analysis. JBI Database System Rev Implement Rep. 2016;14 (7):169-207. doi:10.11124/JBISRIR-2016-003011

2. Zhou L, Xu DY, Sha WG, Shen L, Lu GY. Long non-coding RNA MALAT1 interacts with transcription factor Foxo1 to regulate SIRT1 transcription in high glucose-induced HK-2cells injury. Biochem Biophys Res Commun. 2018;503(2):849-855. doi:10.1016/j. bbrc.2018.06.086

3. Gilbert RE, Cooper ME. The tubulointerstitium in progressive diabetic kidney disease: more than an aftermath of glomerular injury? Kidney Int. 1999;56(5):1627-1637. doi:10.1046/j.1523-1755.199 9.00721.x

4. Chen LL. The biogenesis and emerging roles of circular RNAs. Nat Rev Mol Cell Biol. 2016;17(4):205-211. doi:10.1038/nrm.2015.32

5. Lasda E, Parker R. Circular RNAs: diversity of form and function. RNA. 2014;20(12):1829-1842. doi:10.1261/rna.047126.114

6. Zhou B, Yu JW. A novel identified circular RNA, circRNA_010567, promotes myocardial fibrosis via suppressing miR-141 by targeting TGF-beta1. Biochem Biophys Res Commun. 2017;487(4):769-775. doi:10.1016/j.bbrc.2017.04.044

7. Xia W, Qiu M, Chen R, et al. Circular RNA has_circ_0067934 is upregulated in esophageal squamous cell carcinoma and promoted proliferation. Sci Rep. 2016;6:35576. doi:10.1038/srep35576

8. Wu H, Wu S, Zhu Y, et al. Hsa_circRNA_0054633 is highly expressed in gestational diabetes mellitus and closely related to glycosylation index. Clin Epigenetics. 2019;11(1):22. doi:10.1186/ s13148-019-0610-8

9. Hu W, Han Q, Zhao L, Wang L. Circular RNA circRNA_15698 aggravates the extracellular matrix of diabetic nephropathy mesangial cells via miR-185/TGF-beta1. $J$ Cell Physiol. 2019;234 (2):1469-1476. doi:10.1002/jcp.26959

10. Jin G, Wang Q, Hu X, et al. Profiling and functional analysis of differentially expressed circular RNAs in high glucose-induced human umbilical vein endothelial cells. FEBS Open Bio. 2019;9 (9):1640-1651. doi:10.1002/2211-5463.12709

11. Alebiosu CO, Ayodele OE. The increasing prevalence of diabetic nephropathy as a cause of end stage renal disease in Nigeria. Trop Doct. 2006;36(4):218-219. doi:10.1258/004947506778604797

12. Magee C, Grieve DJ, Watson CJ, Brazil DP. Diabetic nephropathy: a tangled web to unweave. Cardiovasc Drugs Ther. 2017;31(5-6):579-592. doi:10.1007/s10557-017-6755-9

13. Morii T, Fujita H, Narita T, et al. Association of monocyte chemoattractant protein-1 with renal tubular damage in diabetic nephropathy. $J$ Diabetes Complications. 2003;17(1):11-15. doi:10.1016/S10568727(02)00176-9

14. Li D, Kong C, Tsun A, et al. MiR-125a-5p decreases the sensitivity of treg cells toward IL-6-mediated conversion by inhibiting IL-6R and STAT3 expression. Sci Rep. 2015;5:14615. doi:10.1038/ srep14615

15. Brezniceanu ML, Lau CJ, Godin N, et al. Reactive oxygen species promote caspase-12 expression and tubular apoptosis in diabetic nephropathy. J Am Soc Nephrol. 2010;21(6):943-954. doi:10.1681/ ASN.2009030242

16. Abdo S, Lo CS, Chenier I, et al. Heterogeneous nuclear ribonucleoproteins $\mathrm{F}$ and $\mathrm{K}$ mediate insulin inhibition of renal angiotensinogen gene expression and prevention of hypertension and kidney injury in diabetic mice. Diabetologia. 2013;56(7):1649-1660. doi:10.1007/ s00125-013-2910-4
17. Ma J, Li YJ, Chen X, Kwan T, Chadban SJ, Wu H. Interleukin 17A promotes diabetic kidney injury. Sci Rep. 2019;9(1):2264. doi:10.1038/s41598-019-38811-4

18. Lv N, Li C, Liu X, Qi C, Wang Z. miR-34b alleviates high glucose-induced inflammation and apoptosis in human HK-2 cells via IL-6R/JAK2/STAT3 signaling pathway. Med Sci Monit. 2019;25:8142-8151. doi:10.12659/MSM.917128

19. Fu Y, Wang $\mathrm{C}$, Zhang $\mathrm{D}$, et al. Increased TRPC6 expression is associated with tubular epithelial cell proliferation and inflammation in diabetic nephropathy. Mol Immunol. 2018;94:75-81. doi:10.1016/j. molimm.2017.12.014

20. Wang M, Yu F, Wu W, et al. Circular RNAs: a novel type of non-coding RNA and their potential implications in antiviral immunity. Int J Biol Sci. 2017;13(12):1497-1506. doi:10.7150/ ijbs. 22531

21. Liu H, Wang X, Wang ZY, Li L. Circ_0080425 inhibits cell proliferation and fibrosis in diabetic nephropathy via sponging miR-24-3p and targeting fibroblast growth factor 11. J Cell Physiol. 2020;235 (5):4520-4529. doi:10.1002/jcp.29329

22. Yao T, Zha D, Hu C, Wu X. Circ_0000285 promotes podocyte injury through sponging miR-654-3p and activating MAPK6 in diabetic nephropathy. Gene. 2020;747:144661. doi:10.1016/j. gene.2020.144661

23. Liu H, Cheng Y, Xu Y, et al. The inhibition of tumor protein $\mathrm{p} 53$ by microRNA-151a-3p induced cell proliferation, migration and invasion in nasopharyngeal carcinoma. Biosci Rep. 2019;39(10). doi:10.1042/BSR20191357

24. Cheng JL, Zhao H, Yang SG, Chen EM, Chen WQ, Li LJ. Plasma miRNA-122-5p and miRNA-151a-3p identified as potential biomarkers for liver injury among CHB patients with PNALT. Hepatol Int. 2018;12(3):277-287. doi:10.1007/s12072-018-98 71-0

25. Delic D, Eisele C, Schmid R, Luippold G, Mayoux E, Grempler R. Characterization of micro-RNA changes during the progression of type 2 diabetes in Zucker diabetic fatty rats. Int J Mol Sci. 2016;17:5. doi:10.3390/ijms 17050665

26. Liu X, Su X, Xu S, et al. MicroRNA in vivo precipitation identifies miR-151-3p as a computational unpredictable miRNA to target Stat3 and inhibits innate IL-6 production. Cell Mol Immunol. 2018;15 (2):99-110. doi:10.1038/cmi.2017.82

27. Rescher U, Gerke V. Annexins-unique membrane binding proteins with diverse functions. J Cell Sci. 2004;117(Pt 13):2631-2639. doi: $10.1242 /$ jcs. 01245

28. Liu X, Yang G, Fan Q, Wang L. Proteomic profile in glomeruli of type-2 diabetic KKAy mice using 2-dimensional differential gel electrophoresis. Med Sci Monit. 2014;20:2705-2713. doi:10.12659/ MSM.893078

29. Wang YZ, Xu WW, Zhu DY, et al. Specific expression network analysis of diabetic nephropathy kidney tissue revealed key methylated sites. J Cell Physiol. 2018;233(10):7139-7147. doi:10.1002/ jcp. 26638

30. Wang Y, Cheng YS, Yin XQ, Yu G, Jia BL. Anxa2 gene silencing attenuates obesity-induced insulin resistance by suppressing the NF-kappaB signaling pathway. Am J Physiol Cell Physiol. 2019;316(2):C223-C234. doi:10.1152/ajpcell.00242.2018

31. Zhao D, Ge H, Ma B, et al. The interaction between ANXA2 and lncRNA Fendrr promotes cell apoptosis in caerulein-induced acute pancreatitis. J Cell Biochem. 2018. 


\section{Publish your work in this journal}

Diabetes, Metabolic Syndrome and Obesity: Targets and Therapy is an international, peer-reviewed open-access journal committed to the rapid publication of the latest laboratory and clinical findings in the fields of diabetes, metabolic syndrome and obesity research. Original research, review, case reports, hypothesis formation, expert opinion and commentaries are all considered for publication. The manuscript management system is completely online and includes a very quick and fair peer-review system, which is all easy to use. Visit http://www.dovepress.com/testimonials.php to read real quotes from published authors.

Submit your manuscript here: https:/www.dovepress.com/diabetes-metabolic-syndrome-and-obesity-targets-and-therapy-journal 